\title{
Adaptable switching schemes for time-encoded multichannel optical coherence tomography
}

\author{
Andreas Wartak \\ Florian Beer \\ Bernhard Baumann \\ Michael Pircher \\ Christoph K. Hitzenberger
}




\title{
Adaptable switching schemes for time-encoded multichannel optical coherence tomography
}

\author{
Andreas Wartak, ${ }^{a, *}$ Florian Beer, ${ }^{\mathrm{a}, \mathrm{b}}$ Bernhard Baumann, ${ }^{\mathrm{a}}$ Michael Pircher, ${ }^{\mathrm{a}}$ and Christoph K. Hitzenberger ${ }^{\mathrm{a}}$ \\ ${ }^{a}$ Medical University of Vienna, Center for Medical Physics and Biomedical Engineering, Vienna, Austria \\ bienna University of Technology, Institute of Applied Physics, Vienna, Austria
}

\begin{abstract}
We introduce the approach of variable time encoding for multichannel optical coherence tomography (OCT). High-speed fiber optical switches are applied for sequential sample arm switching to enable quasisimultaneous image acquisition from three different orientation angles. In comparison with previous multichannel OCT (using simultaneous sample illumination), time-encoded multichannel OCT has no need for division of illumination power among the respective channels to satisfy laser safety requirements. Especially for ophthalmic applications - in particular retinal imaging, which the presented prototype was developed for-this advantage strongly influences image quality through an enhanced sensitivity. Nevertheless, time encoding comes at the cost of a decrease in imaging speed due to sequential channel illumination. For the typical multichannel OCT modality Doppler OCT, this results in a reduction of the maximum unambiguously determinable Doppler velocity. However, we demonstrate that this drawback can be overcome by adaptation of the illumination channel switching scheme. Thus, a re-extension of the maximum unambiguously determinable Doppler frequency to the full A-scan rate of the tunable light source is presented. The performance of the technique is demonstrated by flow phantom experiments and measurements of retinal blood flow in the eyes of healthy human volunteers. $\odot$ The Authors. Published by SPIE under a Creative Commons Attribution 3.0 Unported License. Distribution or reproduction of this work in whole or in part requires full attribution of the original publication, including its DOI. [DOI: 10.1117/1.JBO.23.5.056010]
\end{abstract}

Keywords: optical coherence tomography; optical switching; Doppler; ophthalmology; retinal imaging.

Paper 180053PR received Jan. 24, 2018; accepted for publication Apr. 30, 2018; published online May 24, 2018.

\section{Introduction}

Tackling the quantification of flow in living tissue (primarily blood flow), optical coherence tomography's (OCT) functional extension Doppler OCT (DOCT) is in principle limited to the determination of the axial velocity component. ${ }^{1}$ In the quest for absolute velocity measurements, various approaches attempted to determine the missing link-the Doppler angle in between illumination direction and direction of movement of the scatterer. Some approaches used additional structural data to externally retrieve information about flow orientation. ${ }^{2-4}$ Others integrated an additional illumination channel to obtain simultaneous axial velocity component measurements from two different observation angles including advanced alignment strategies. ${ }^{5,6}$ Again others combined both the additional structural data and the dual-channel approach. ${ }^{7,8}$ Nevertheless, multichannel OCT only enabled first absolute velocity measurements without any prior knowledge of flow orientation by increasing the number of individual linearly independent sampling directions to three. ${ }^{9}$

In ophthalmology-OCT's most prominent field of application-strict laser safety requirements have to be met. ${ }^{10}$ To achieve highest sensitivity, illumination powers in OCT usually range just below the maximum permissible exposure (MPE) limits. Thus, multichannel OCT instruments, focusing their multiple beams onto a mutual target spot, suffer from reduced sensitivity due to necessary division of illumination power among their respective channels (e.g., 95-dB per channel at

*Address all correspondence to: Andreas Wartak, E-mail: andreas.wartak@ meduniwien.ac.at
20-kHz A-scan rate). ${ }^{9}$ To avoid this division of illumination power, our group recently introduced a further advancement in multichannel DOCT, with the design of a prototype employing an active-passive channel approach using path-length encoding (APPLE DOCT). ${ }^{11}$ This instrument featured only a single active illumination channel (with full illumination power) while simultaneously probing the sample with two passive detection-only channels from two additional directions. However, even though the active-passive principle provided promising results and its capability to determine the potential biomarker total retinal blood flow (RBF) was demonstrated in healthy volunteers, recoupling of the backscattered light into the passive channels proved much more sensitive to optical aberrations introduced by the sample than active recoupling. Already moderate optical aberrations resulted in a decrease of image quality, which highly complicated imaging of the diseased eyes of patients. Only an actively controlled recoupling/ realignment process of the passive channels during the measurement, as proposed previously ${ }^{12}$ - similar to the feedback loops employed in adaptive optics OCT-would potentially solve this issue.

In this paper, we introduce a concept of multichannel OCT, which combines the advantages of both our previously published multichannel instruments: (i) active recoupling of all three illumination channels, ${ }^{9}$ (ii) full illumination power for each channel ${ }^{11}$ (enabling sensitivities of 96- to $97-\mathrm{dB}$ per channel at $100 \mathrm{kHz}$ ). To satisfy laser safety limits, ${ }^{10}$ the multiple active channels are employed in temporally consecutive order using the technique of optical switching. ${ }^{13}$ The presented timeencoded multichannel OCT prototype incorporates three high- 
speed fiber optical switches enabling signal recording from three linearly independent orientations quasisimultaneously.

Optical switching, in general, can be achieved by mechanical, acousto-opitcal, magneto-optical, or electro-optical means. Although the concept of electro-optical switching originates from telecommunications, the method has recently been applied to the field of OCT as well. High-speed fiber optical switches were previously reported for the removal of the autocorrelation term, ${ }^{14}$ extension of image range, ${ }^{15}$ parallelization of detection, ${ }^{16,17}$ sweep buffering ${ }^{18}$ as well as sensitivity enhancement through balanced detection. ${ }^{19}$ These approaches integrated optical switches right after the light source, within the reference arm or before the detection unit of their respective interferometers. However, the installment of optical switches within the sample arm for the purpose of multichannel OCT outlines a new concept.

Time encoding, in principle, decreases the imaging speed as consecutive A-scans are divided among the respective channels. For DOCT, a reduction in imaging speed (equivalent to a temporal increase of the sampling interval- $-T$ ) consequently implicates a decrease in the maximum unambiguously determinable axial velocity component $-\vec{v}_{\max }(T)=\frac{\lambda_{0}}{4 n T}{ }^{1}{ }^{1} 2 \pi$-phaseambiguity artifacts in the phase-difference images (Doppler tomograms), called phase wrapping, are generated at velocities exceeding $\vec{v}_{\max }(T)$. Phase wrapping artifacts typically heavily influence results of velocity measurements. Even though phase unwrapping is used in various optical imaging modalities ${ }^{20,21}$ and also already found its way into OCT and DOCT postprocessing algorithms, ${ }^{22-25}$ phase wrapping can still be very challenging (mostly due to the presence of phase noise) and thus should be avoided. ${ }^{26-28}$

To overcome the drawback of the reduced accessible ambiguity-free velocity range for DOCT, additional illumination channel switching schemes are introduced in this article. This adaptability in terms of sampling intervals provides a considerable extension of the maximum unambiguously determinable Doppler frequency. We demonstrate the capabilities of the proposed method in vitro by flow phantom measurements as well as in vivo by RBF measurements in the eyes of healthy human volunteers.

\section{Methods}

\subsection{Experimental Setup}

A sketch of the time-encoded multichannel OCT prototype is shown in Fig. 1. Similar to our previous APPLE D-OCT

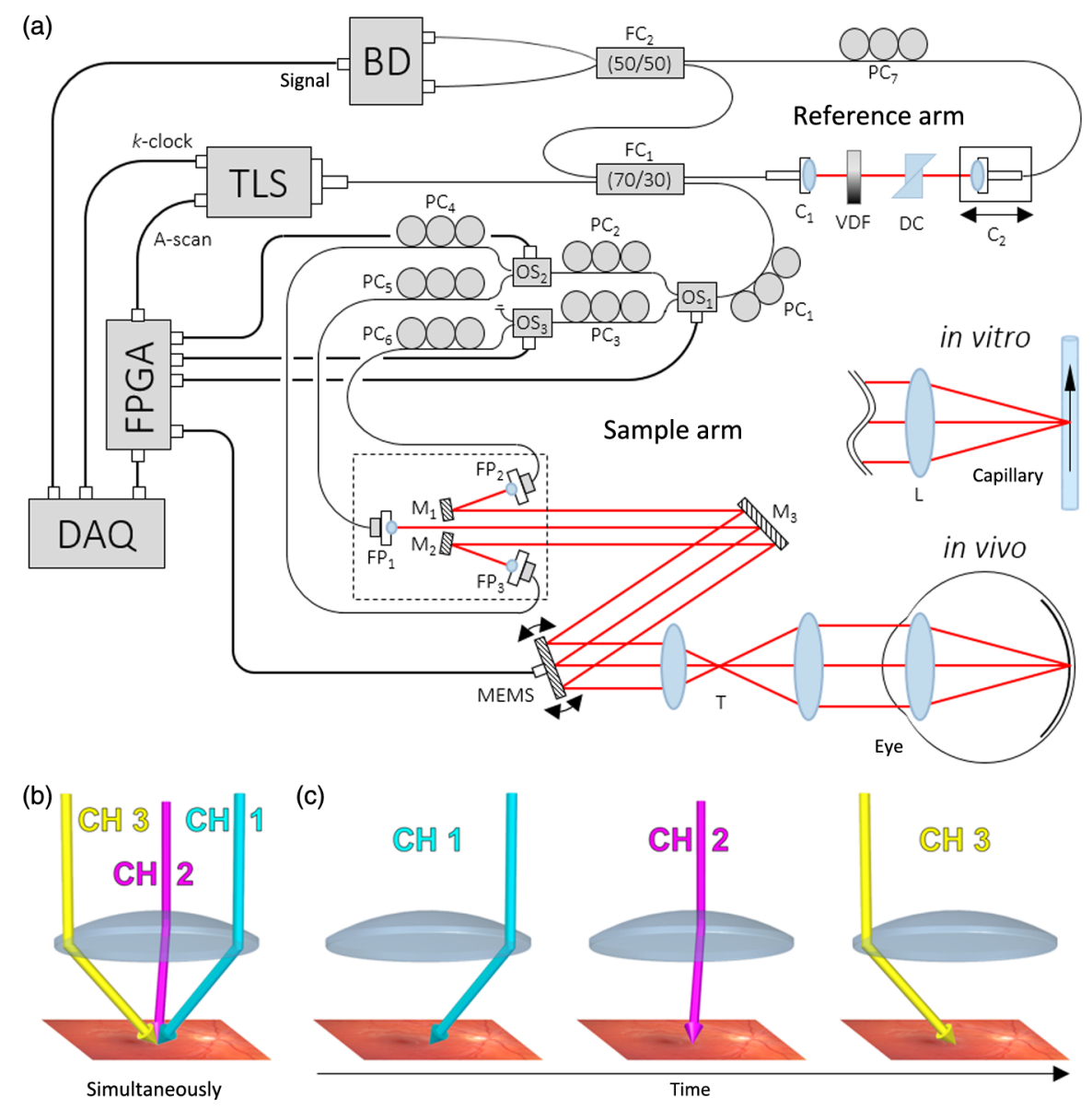

Fig. 1 (a) Sketch of the time-encoded three-channel OCT setup: TLS, tunable light source; FC, fiber coupler; PC, polarization control paddles; C, collimator; VDF, variable density filter; $D C$, dispersion compensation; OS, $1 \times 2$ fiber optical switch; FP, fiber port collimator; M, mirror; MEMS, 2-D scanning mirror; $\mathrm{T}$, telescope; L, lens; BD, balanced amplified photodetection unit; FPGA, field programmable gate array; and DAQ, data acquisition board. (b) Simultaneous illumination: beam geometry as of Ref. 9 (division of power among three beams). (c) Sequential (or quasisimultaneous) illumination: beam geometry of timeencoded three-channel OCT prototype (no division of power). 
setup, ${ }^{11}$ this setup is based on a single-mode (SM) fiber-based Mach-Zehnder interferometer. The light emitted by the wavelength-tunable laser source (TLS; Axsun Technologies, Inc.; 1060 Swept Laser Engine; center wavelength: $\lambda_{0}=1045 \mathrm{~nm}$; bandwidth: $\Delta \lambda=100 \mathrm{~nm}$; sweep/A-scan rate: $f=100 \mathrm{kHz}$; duty cycle: $d=\sim 50 \%$ ) was directly coupled into a fiber coupler ( $\mathrm{FC}_{1}$; all FCs: AC Photonics, Inc.). $\mathrm{FC}_{1}$ divided the light at a split ratio of $70 / 30$ into reference $(70 \%)$ and sample beam (30\%).

In the sample arm, the light was guided through the centerpiece of the whole setup - an assembly of three high-speed optical $1 \times 2$ switches $\left(\mathrm{OS}_{1-3}\right.$; Agiltron, Inc.; NanoSpeed ${ }^{\mathrm{ms}} 1 \times 2$ fiberoptic switch; $500 \mathrm{kHz}$ repetition rate NanoSpeed ${ }^{\mathrm{TM}}$ switch driver). The switch drivers enable an increase in the maximum repetition/switch rate from 5 to $500 \mathrm{kHz}$ (stated by the manufacturer)-which is five-times faster than the A-scan rate of the TLS. The three switches were arranged in a way that the two output ports of $\mathrm{OS}_{1}$ were directly connected to the input ports of $\mathrm{OS}_{2}$ and $\mathrm{OS}_{3}$. However, only three of the combined four output ports of $\mathrm{OS}_{2}$ and $\mathrm{OS}_{3}$ were employed to enable three-channel switching. In principle, two optical $1 \times 2$ switches would have been sufficient to enable three-channel switching. $\mathrm{OS}_{3}$ was only integrated for necessary dispersion matching and was not operated. To control the incoming and outgoing polarization state of the light, and thus enable individual polarization matching with regard to the polarization state of the reference arm light for each sample arm independently, polarization control paddles $\left(\mathrm{PC}_{1-6}\right)$ were integrated in front and after each switch. A field programmable gate array (FPGA) module, clocked by the A-scan trigger exiting the TLS, provided the voltage signals driving the optical switches.

Three of the four output ports of $\mathrm{OS}_{2}$ and $\mathrm{OS}_{3}$ were directly connected to the beam-alignment unit (cf. dotted box in Fig. 1). In comparison with our previously reported multichannel setups,,${ }^{9,11}$ the single block brass mount in combination with three miniature fiber collimators (MC) was replaced, which had only provided a beam separation of $4.4 \mathrm{~mm}$ and thus caused spatial restrictions. After an initial parallel alignment of the MCs, any further adjustment was rather impractical. Especially, within the time-frame of an in vivo measurement this was impossible. The improved beam-alignment unit incorporated three FiberPort collimators $\left(\mathrm{FP}_{1-3}\right.$; Thorlabs, Inc.; PAFA-X-4-CAchromatic FiberPort) as well as two D-shaped gold mirrors $\left(\mathrm{M}_{1-2}\right.$; diameter: $\left.12.7 \mathrm{~mm}\right)$. The separation distances between the three collimated beams (triangle configuration ${ }^{9}$ ) could be decreased to fit all of them onto the scanning unit. Thus, the facet prism telescope (FPT) that was integrated in our previously reported multichannel setups ${ }^{11,29,30}$ could be omitted while a narrow enough beam separation to enable sharing of all optical elements in the sample beam path among all three channels could be provided.

From a stationary mirror $\left(\mathrm{M}_{3}\right)$, the collimated beams were directed onto a dual-axis gimbal-less MEMS mirror (Mirrorcle Technologies, Inc.; diameter: $3.6 \mathrm{~mm}$; mechanical tilt angle ( $x$ - and $y$-axes): $-6.5 \mathrm{deg}$ to $6.5 \mathrm{deg}$ ), which was employed for 2-D scanning. The mirror $\mathrm{M}_{3}$ introduced a fairly small angle of incidence onto the scanning unit to minimize geometrical scanning distortions. ${ }^{31}$ The scanning mirror was synchronized to the TLS using its A-scan trigger and the FPGA module. Being reflected from the scanning mirror, the light was guided through a 50/80 telescope (T) to increase the beam separation distances from $\sim 1.8$ to $\sim 2.9 \mathrm{~mm}$ (determined by a beam profile measurement). Considering beam diameters of $\sim 0.8 \mathrm{~mm}$ (corresponding to a transverse resolution of $\sim 32 \mu \mathrm{m}$ for retinal imaging), the minimally required pupil aperture was calculated to be $\sim 4.2 \mathrm{~mm}$. Therefore, at low light conditions no mydriatics were needed to dilate the subject's pupil for in vivo measurements. However, in case of a smaller pupil, subject motion needed to be considered in terms of blocking one of the three collimated beams at the iris. The $\sim 2.9-\mathrm{mm}$ separation distances were empirically found to provide a good compromise to enable substantially different inclination of the measurement beams with respect to each other (a prerequisite for multichannel DOCT-cf. Ref. 9) while avoiding blocking of the beams by the irises of our healthy subjects. Finally, the beams were focused onto a mutual spot on the retina by the refractive optics of the human eye. For flow phantom measurements, an additional focusing lens ( $\mathrm{L} ; f=30 \mathrm{~mm}$ ) was used instead.

The three time-encoded beams were aligned along the corners of an equilateral triangle as in Ref. 9. However, contrary to the geometry used in Ref. 9, the three active channels did not illuminate the sample simultaneously but in temporal succession-cf. Figs. 1(b) and 1(c).

Due to sequential illumination (and in comparison with Refs. 9, 29, and 30), no division of beam power among the three channels was required to meet the laser safety standards. According to the MPE limits, ${ }^{10}$ ocular exposures were $1.9 \mathrm{~mW}$ or less (same power also for in vitro experiments).

In the reference arm, the light was coupled out via a fiber collimator $\left(C_{1}\right)$. A variable density filter (VDF) was used for optical power adjustments and two identical N-BK7 glass prisms (DC) were integrated to compensate for dispersion mismatch between reference and sample arms. A second collimator $\left(C_{2}\right)$ (mounted on a translation stage) coupled the light into another SM fiber. The light traveling through the reference arm was finally recombined with the backscattered light from the sample arm at a 50/50 $\mathrm{FC}\left(\mathrm{FC}_{2}\right)$. To enable polarization matching between all three sample arm channels and the reference arm, polarization control paddles $\left(\mathrm{PC}_{7}\right)$ were used.

Both outputs of $\mathrm{FC}_{2}$ were connected to a balanced amplified photodetection unit (BD; Thorlabs, Inc.; PDB 460C), which detected the interference patterns with a bandwidth of $200 \mathrm{MHz}$. The signal was digitized with a variable frequency of up to $350 \mathrm{MHz}$ (max. $k$-clock frequency) at 12-bit resolution via a PCIe data acquisition (DAQ) board (AlazarTech, Inc.; ATS9350). To sample the spectral data at equal intervals in $k$-space, the TLS's integrated $k$-clock was utilized.

Using a mirror as a sample, exposures of $1.9 \mathrm{~mW}$ resulted in a measured sensitivity of $\sim 96-$ to $97-\mathrm{dB}$ per channel. The instrument was operated close to the shot noise limit that was calculated to be $98.8 \mathrm{~dB}$. The lower sensitivity of the presented system in comparison with other TLS systems can be mostly attributed to transmission losses in the OSs. Roughly, 25\% single-pass power loss was experienced for light transmitted through one OS. The sensitivity roll-off was determined to be $\sim 1.5 \mathrm{~dB}$ for the imaging range of $3.7 \mathrm{~mm}$ in air. The axial resolution was measured to be $\sim 7 \mu \mathrm{m}$-corresponding to $\sim 5 \mu \mathrm{m}$ within the retina (refractive index: 1.4 ).

\subsection{Measurement Protocol}

For the in vivo investigations in three healthy volunteers (age range: 28 to 34 ), informed consent was obtained prior to each measurement. The study was in agreement with the tenets 
of the Declaration of Helsinki and was approved by the institutional ethics committee. Before the measurement, the axial eyelength of each subject was determined by partial coherence interferometry (Zeiss; IOL Master). ${ }^{32}$ Subject positioning, target fixation as well as measurement surveillance were similar to previously published methods. ${ }^{11}$

For both in vitro and in vivo experiments, only linear scanning patterns consisting of consecutive B-scans at the same sample position were applied. For the in vitro scenario, datasets of 3072 A-scans $\times 50$ B-scans were recorded, whereas for the in vivo scenario the datasets contained 8190 A-scans $\times 30$ Bscans. For both cases also reduced datasets of only 512 and 1365 A-scans per B-scan were acquired.

\subsection{Doppler Optical Coherence Tomography Data Processing}

The DOCT postprocessing steps are similar to those published previously ${ }^{9,11,29,30}$ and will thus only be covered briefly.

Mean spectrum subtraction, numerical dispersion compensation, and discrete FFT - the standard Fourier domain (FD-) OCT data processing steps - were performed on the acquired raw data at first. The second step was the calculation of the phase difference or Doppler tomograms. Here, only pixels above an empirically chosen intensity threshold $(\sim 2.5$-times the intensity noise level) were considered. The phase shifts introduced by the 2-D scanner (constant offset due to linear scanning pattern) were compensated for using the same histogram-based method as in Ref. 11 for both the in vitro and in vivo scenarios. In a third step, Doppler tomograms were averaged in complex space to (i) obtain better image quality in the in vitro case and (ii) to additionally average the pulsatile characteristic of retinal vessels out in the in vivo scenario. Due to stable imaging conditions, without sample movement, no image registration needed to be applied for the in vitro experiment. In the in vivo case, the same manual vessel registration method as described in Refs. 11 and 30 was applied. Only B-scans within one cardiac cycle were averaged (five to seven B-scans of 8190 A-scans for a subject heart rate of $\sim 55$ to $75 \mathrm{bpm}$ ). The systolic peaks in the velocity profiles of arteries helped to determine the exact number of B-scans per individual cardiac cycle.

To retrieve the velocity information from a Doppler tomogram, the obtained phase shift between successive A-scans $\Delta \varphi$ is related to the axial velocity component $\vec{v}_{a x}$ as follows: ${ }^{1}$

$\vec{v}_{a x}=\frac{\Delta \varphi \lambda_{0}}{4 \pi n T}$

where $\lambda_{0}$ denotes the central wavelength of the emitted light, $n$ is the refractive index of the medium, and $T$ is the time delay in between successive A-scans. To obtain the absolute velocity $\vec{v}_{\text {abs }}$ of the moving particle, the Doppler angle $\alpha$ needs to be known

$\left|\vec{v}_{\mathrm{abs}}\right|=\left|\frac{\vec{v}_{a x}}{\cos (\alpha)}\right|$.

In general, the determination of the Doppler angle requires additional information about the orientation of movement. Our group previously showed that by the use of three individual sampling beams probing the target from three linearly independent orientations, the absolute velocity $\vec{v}_{\text {abs }}$ can be determined without any prior knowledge on the orientation of movement. ${ }^{9}$ Thus, only the exact spatial orientations of the three sampling beams have to be determined to retrieve $\vec{v}_{\text {abs }}$. This requires a measurement of the beam separation distances (using a beam profiler in the pupil plane) as well as of the focal length of the respective refractive optics. Using these values, a so-called beam geometry matrix (BGM) can be calculated that eventually is multiplied with the three mean velocity components from the velocity profiles obtained from the vessel per channel. ${ }^{11}$ As only a rather small scanning angle $(<2.5 \mathrm{deg})$ was applied, the systematic errors due to alteration of the BGM while scanning could be neglected, ${ }^{9,11}$ and there was no need for adapting the BGM.

\subsection{Switching Schemes}

The integration of high-speed optical switches enables a variety of possible switching schemes. For the presented RBF investigations, three different switching schemes are demonstrated. Their designation as well as their main specifications are shown in Table 1. The timing diagrams for the triggering of the optical switches are shown in Figs. 2(a)-2(c), respectively.

The 1-2-3-1 scheme directs the light for the duration of exactly one A-scan $(10 \mu \mathrm{s})$ through one channel (1) before switching to the next channel (2) for the following spectral sweep of the TLS. The third sweep is directed to the third channel (3) before it is again the first channel's turn (1). Only one third of the acquired A-scans can be accounted for each individual channel that is equivalent to a reduced sampling frequency. The imaging speed of each channel is reduced to a third of the original sweep rate of $100 \mathrm{kHz}$. Thus, the maximum unambiguously determinable Doppler frequency is reduced to $33.3 \mathrm{kHz}$. However, phase shifts obtained in RBF investigations may exceed Doppler frequencies of $33.3 \mathrm{kHz}$, which would cause phase wrapping artifacts for the 1-2-3-1 scheme. Thus, the following two switching schemes were chosen to regain a maximum Doppler frequency of $100 \mathrm{kHz}$.

The 11-22-33-11 scheme is similar to the 1-2-3-1 scheme but records always two consecutive A-scans per channel (switching interval of $20 \mu \mathrm{s}$ ). Consequently, the maximum unambiguously determinable Doppler frequency is re-extended to $100 \mathrm{kHz}$. However, when calculating the Doppler tomogram, only every sixth phase-difference A-scan contributes to the final 2-D phase-difference image at $100 \mathrm{kHz}$. Thus, regaining the full dynamic velocity range comes at the cost of limited sampling.

Table 1 Summary of the basic specifications of the three introduced channel switching schemes: inter-A-scan (1-2-3-1), double inter-Ascan (11-22-33-11), and intra-A-scan (123-123).

\begin{tabular}{|c|c|c|c|}
\hline Designation & $\begin{array}{l}\text { Inter-A- } \\
\text { scan }\end{array}$ & $\begin{array}{l}\text { Double inter- } \\
\text { A-scan }\end{array}$ & $\begin{array}{l}\text { Intra-A- } \\
\text { scan }\end{array}$ \\
\hline Abbreviation & $1-2-3-1$ & $11-22-33-11$ & 123-123 \\
\hline Imaging speed [kHz] & 33.3 & 16.7 & 100 \\
\hline $\begin{array}{l}\text { Maximum unambiguously } \\
\text { determinable Doppler } \\
\text { frequency }[\mathrm{kHz}]\end{array}$ & 33.3 & 100 & 100 \\
\hline $\begin{array}{l}\text { Doppler tomogram A-scan } \\
\text { number [\%] }\end{array}$ & 33.3 & 16.7 & 100 \\
\hline Axial resolution [\%] & 100 & 100 & 33.3 \\
\hline
\end{tabular}




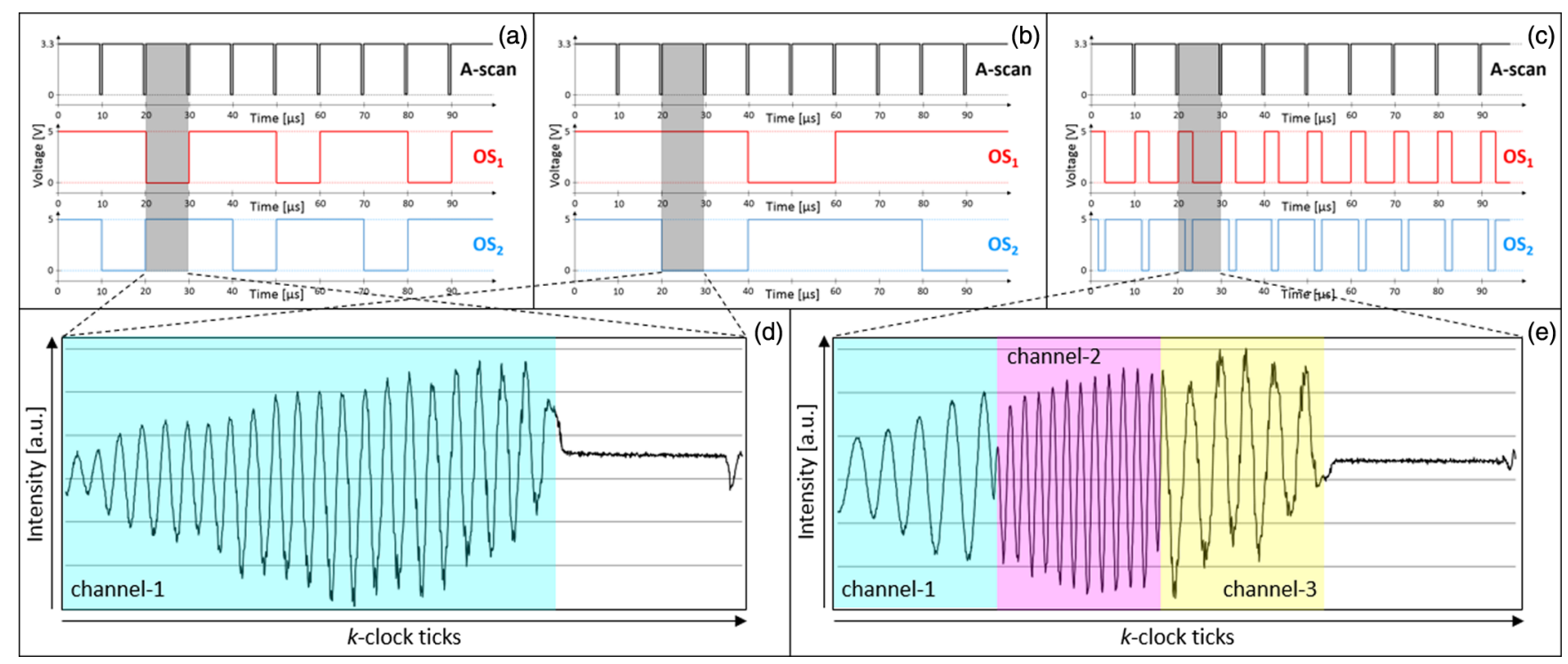

Fig. 2 Timing diagrams of optical switching schemes: (a) inter-A-scan scheme (1-2-3-1): switch triggering and synchronization to the TLS via the FPGA for three channels. Black: A-scan trigger from TLS ( 0 to $3.3 \mathrm{~V} \mathrm{TTL} \mathrm{signal).} \mathrm{Red:} \mathrm{Trigger} \mathrm{signal} \mathrm{for} \mathrm{OS1} \mathrm{(0} \mathrm{to} 5 \mathrm{~V} \mathrm{TTL} \mathrm{signal).} \mathrm{Blue:} \mathrm{trigger} \mathrm{signal} \mathrm{for}$ OS2 (0 to 5 V TTL signal). (b) Double inter-A-scan scheme (11-22-33-11). (c) Intra-A-scan scheme (123-123). (d) Modulated balanced spectrum of channel-1 for schemes 1-2-3-1 and 11-22-33-11. (e) Modulated balanced spectrum of channels 1, 2, 3 for scheme 123-123.

The 123-123 scheme differs from the aforementioned two schemes by even shorter switching intervals that introduce channel switching within one spectral sweep. By application of this intra-A-scan switching scheme, every sweep is divided into three equal temporal sections of $\sim 1.8-\mu$ s duration. The optical switches are thus operated at $\sim 560 \mathrm{kHz}$ (which is slightly above the specified maximum repetition/switch rate of $500 \mathrm{kHz}$ ) to enable three temporally equal sections per sweep. No impacts on the performance were observed when the optical switches were operated $60 \mathrm{kHz}$ above their specified maximum repetition/ switch rate. Figure 2 shows modulated balanced spectra for the 1-2-3-1 and 11-22-33-11 scheme-cf. Fig. 2(d)—as well as for the 123-123 scheme-cf. Fig. 2(e). The differences in the modulation frequencies of the three temporal sections in Fig. 2(e) arise due to slight path-length differences among the three illumination channels. Intra-A-scan switching restores an imaging speed of $100 \mathrm{kHz}$ per channel as well as a maximum unambiguously determinable Doppler frequency of $100 \mathrm{kHz}$.

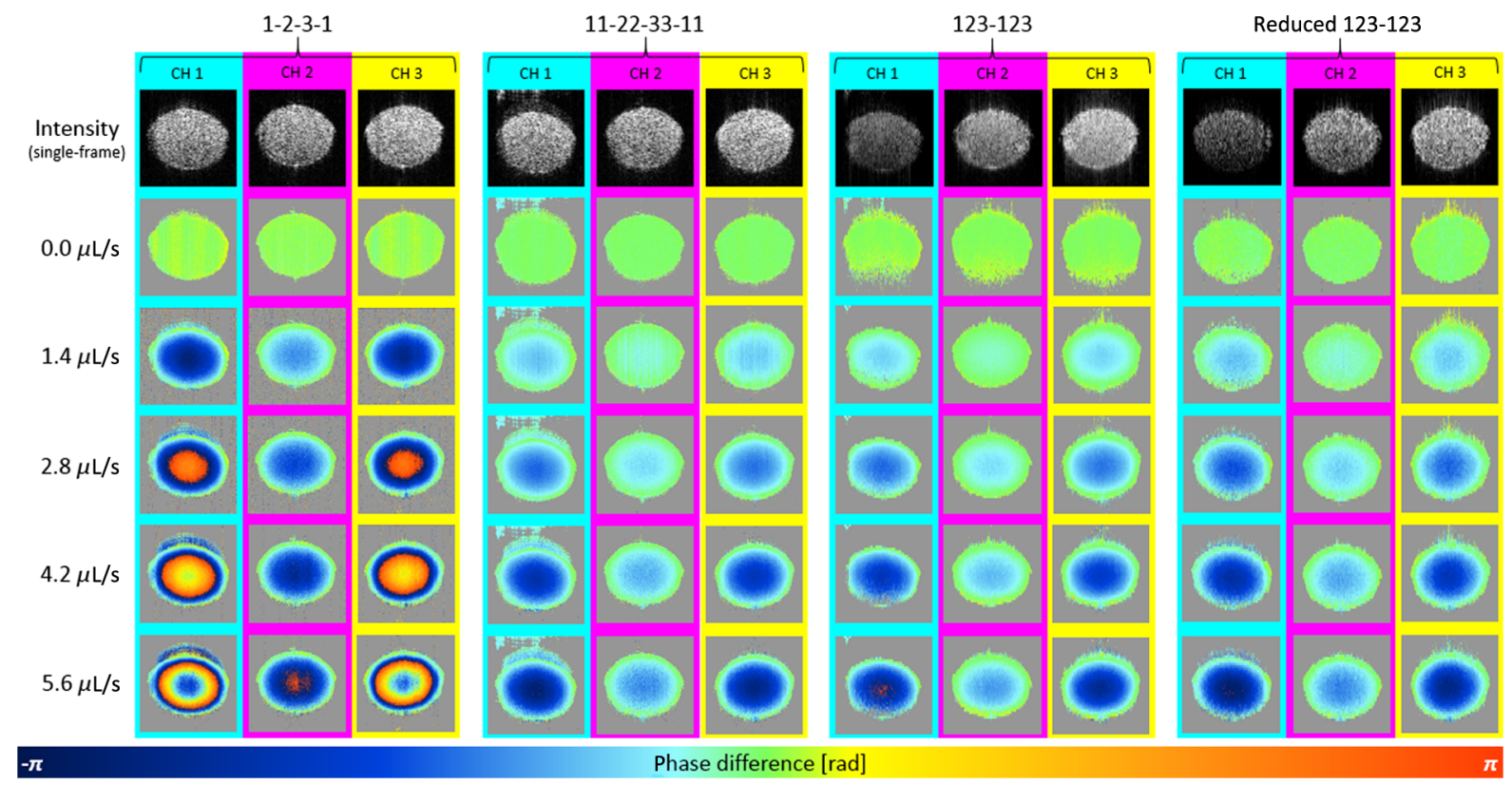

Fig. 3 Imaging results of the capillary measurements for the different illumination channels (channel-1cyan, channel-2-magenta, and channel-3-yellow) and switching schemes (1-2-3-1, 11-22-33-11, 123123 , and reduced 123-123). Varying injection pump flow velocities ( 0.0 to $5.6 \mu \mathrm{L} / \mathrm{s})$ at constant angles of rotation ( $\delta=95 \mathrm{deg}$ and $\rho=5 \mathrm{deg}$ ). In comparison with the other switching schemes, phase wrapping artifacts occur already at $2.8 \mu \mathrm{L} / \mathrm{s}$ for the 1-2-3-1 scheme (Doppler tomograms 20-times averaged). 
Also, three- (six-) times as many A-scans can be evaluated for the Doppler tomograms in comparison with the 1-2-3-1 (11-2233-11) switching scheme. Nevertheless, these benefits come at the cost of a reduced axial resolution, which is limited to about a third of the original value. In a 2-D image, the gain of transversal pixels is thus compensated by the loss in axial direction.

\section{Results}

In the following two subsections, the different switching schemes will be evaluated regarding their usefulness for measurements of absolute velocity of RBF. In a first step, flow phantom measurements were conducted. In a second step, retinal vessels in the eyes of three healthy volunteers were evaluated.

\subsection{In Vitro Experiments Using a Flow Phantom}

A medical injection pump (MGVG; Combimat) was used to perfuse the flow phantom-a glass capillary with an inner diameter of $300 \mu \mathrm{m}$ - by a highly scattering dilution of milk and water (ratio 1:1). The capillary was mounted to allow alignment along three degrees of freedom to demonstrate reconstruction of the absolute $3-\mathrm{D}$ velocity vector independently of flow

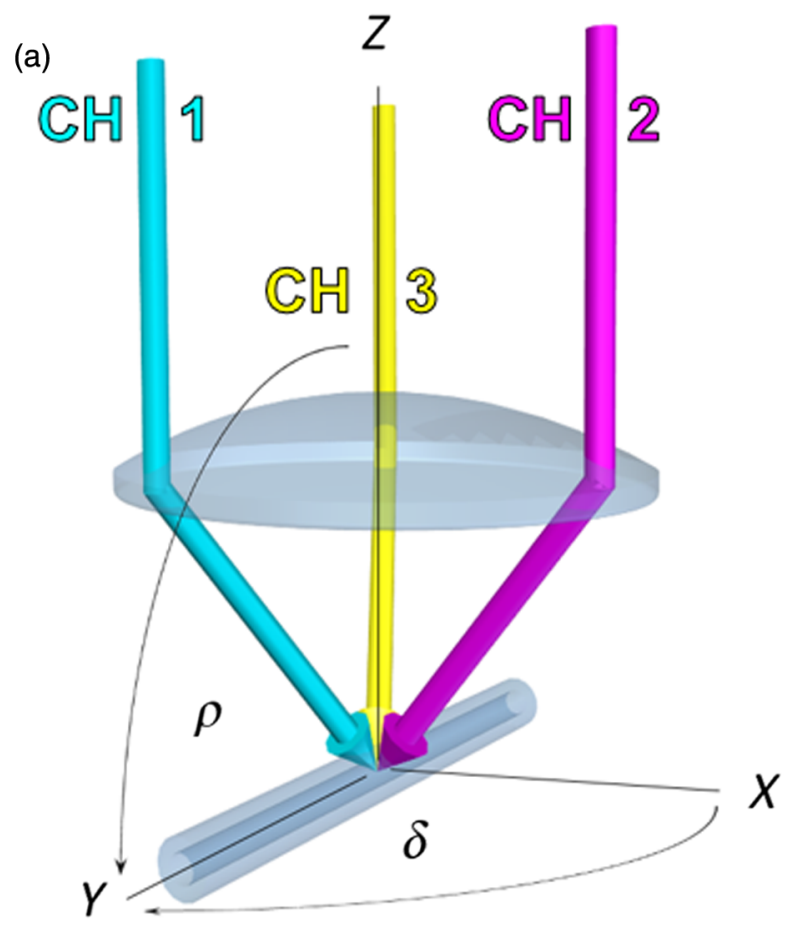

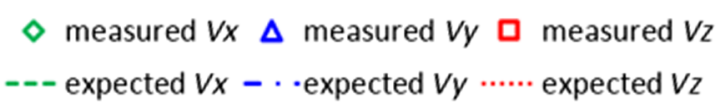

$1-2-3-1$

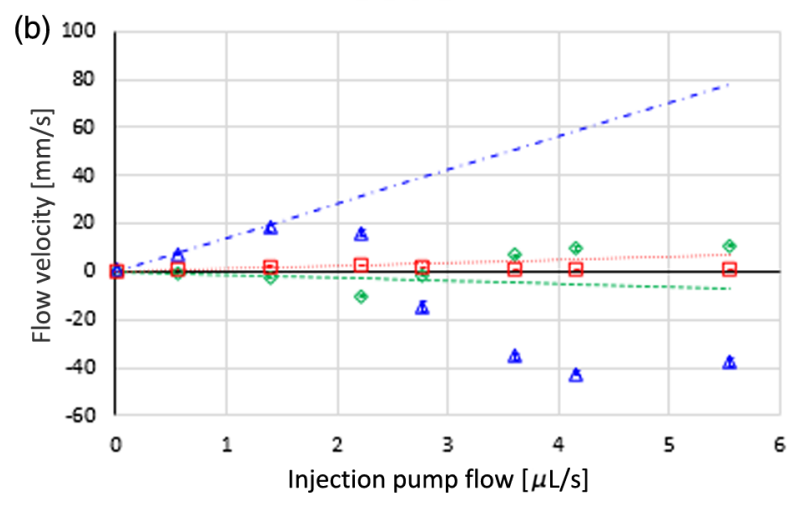

$11-22-33-11$

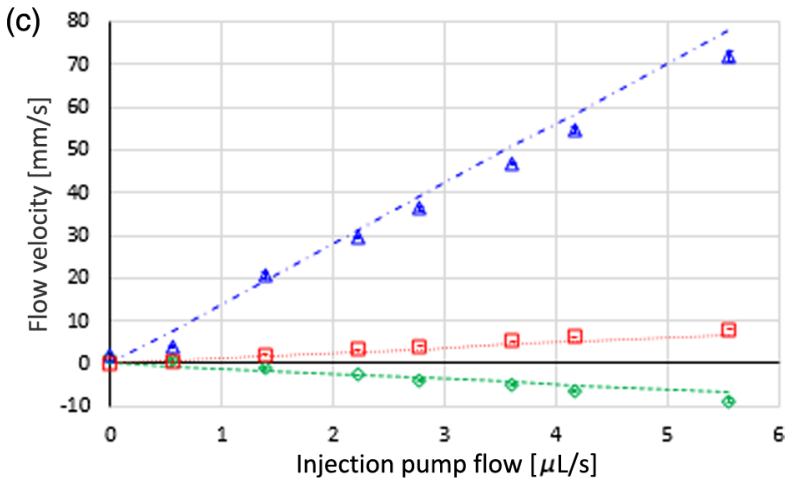

$123-123$

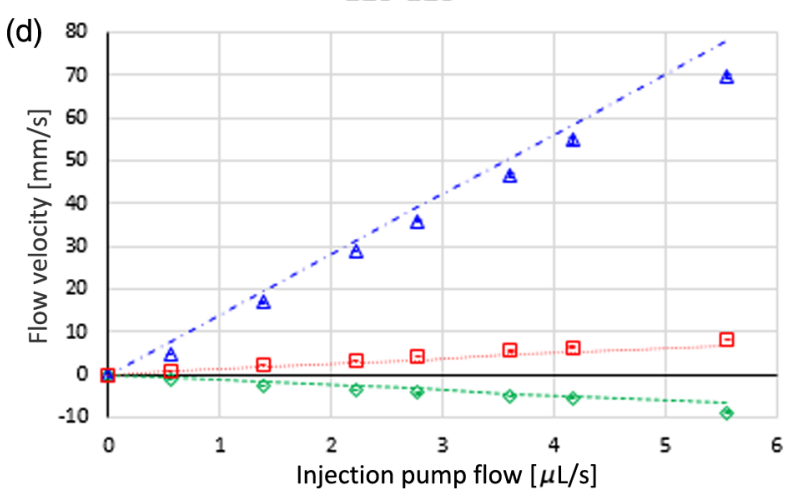

reduced $123-123$

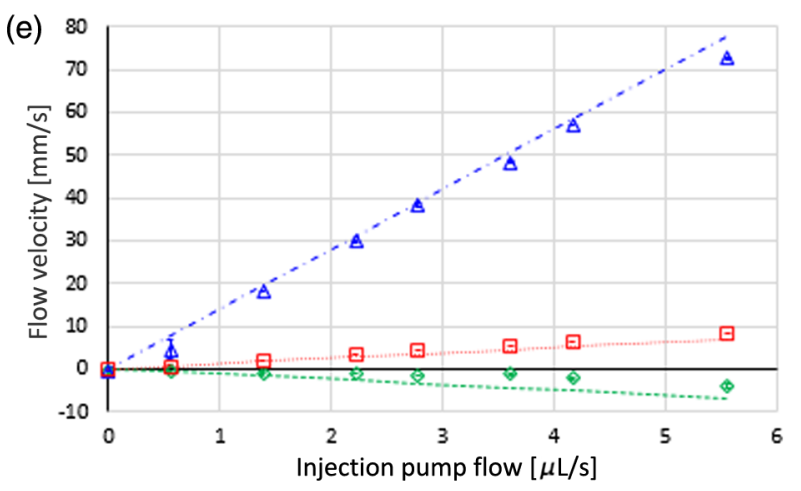

Fig. 4 Quantitative capillary measurement results for the different switching schemes: (a) Illustration of the three-channel illumination beam geometry for the flow phantom measurements. The perfused glass capillary could be rotated with regard to the optical axis along the angle $\delta$ (orthogonal plane) and the angle $\rho$ (parallel plane). (b) 1-2-3-1, (c) 11-22-33-11, (d) 123-123, and (e) reduced 123-123. Expected versus measured velocity vector components $V_{x}, V_{y}$, and $V_{z}$ (20-times averaged) for varying injection pump flows ( 0.0 to $5.6 \mu \mathrm{L} / \mathrm{s})$ at constant angles of rotation $(\delta=95 \mathrm{deg}$ and $\rho=5 \mathrm{deg})$. All values are mean velocity values over cross-sectional profile. Error bars indicate \pm one STD. 
orientation. The in vitro measurement beam geometry including an indication of the two angles of rotation in the plane orthogonal $(\delta)$ and parallel $(\rho)$ to the optical axis can be observed in Fig. 4(a).

In Fig. 3, imaging results of the flow phantom measurements are shown. Four image blocks using different switching schemes are positioned next to each other, containing images from every one of the three illumination channels each. The first row shows single-frame intensity images, whereas the further rows show 20-times averaged Doppler tomograms acquired at changing pump velocities $(0.0$ to $5.6 \mu \mathrm{L} / \mathrm{s})$. The rotational angles were kept constant during the course of this measurement ( $\delta=95 \mathrm{deg}$ and $\rho=5 \mathrm{deg}$ ). The switching scheme of the fourth block-labeled "reduced 123-123"-has not been described previously. This scheme is identical to the 123-123 scheme while recording only a reduced number of A-scans per B-scan (factor of six). This scheme is used to enable a comparison between the 11-22-33-11 and the 123-123 scheme in terms of speed of acquisition (both yield a maximum unambiguously determinable Doppler frequency of $100 \mathrm{kHz}$ ). For the same acquisition time, the 123-123 scheme provides sixtimes as many A-scans for each Doppler tomogram (at reduced axial resolution) as the 11-22-33-11 scheme. Thus, the reduced 123-123 scheme allows for six-times shorter acquisition time at equal sampling frequency to the 11-22-33-11 scheme.
The imaging results of Fig. 3 clearly demonstrate that the 11-22-33-11, the 123-123, and the reduced 123-123 switching scheme enable acquisition of phase wrapping artifact free Doppler tomograms at higher pump velocities than the 1-2-31 scheme, where obvious phase wrapping artifacts appear in channel-1 and channel-3 already at $2.8 \mu \mathrm{L} / \mathrm{s}$.

Although it does not change the measurement results here, a clear sensitivity penalty in comparison with the other two channels can be observed for channel-1 of the 123-123 and the reduced 123-123 scheme. The origin of this issue, which is already apparent when considering the reduced modulation amplitude in the first third of the spectrum presented in Fig. 2(e), is at least partly due to a wavelength dependence/ imbalance of some of the instrument's components (e.g., fiber couplers).

The quantitative evaluation of the Doppler tomograms presented in Fig. 3 is shown in Figs. 4(b)-4(e). Here, the measured velocity components in $x$-, $y$-, and $z$-directions $\left(V_{x}, V_{y}, V_{z}\right)$ are plotted over the expected ones. The indicated values represent mean velocity values over the parabolic shaped velocity profile. The measured values are in good agreement with the expected values for the 11-22-33-11, 123-123, and reduced 123-123 scheme. As soon as the phase wrapping artifacts occur (at a flow velocity of $\sim 2 \mu \mathrm{L} / \mathrm{s}$ ), the 1-2-3-1 scheme no longer provides reliable results without the use of phase unwrapping.
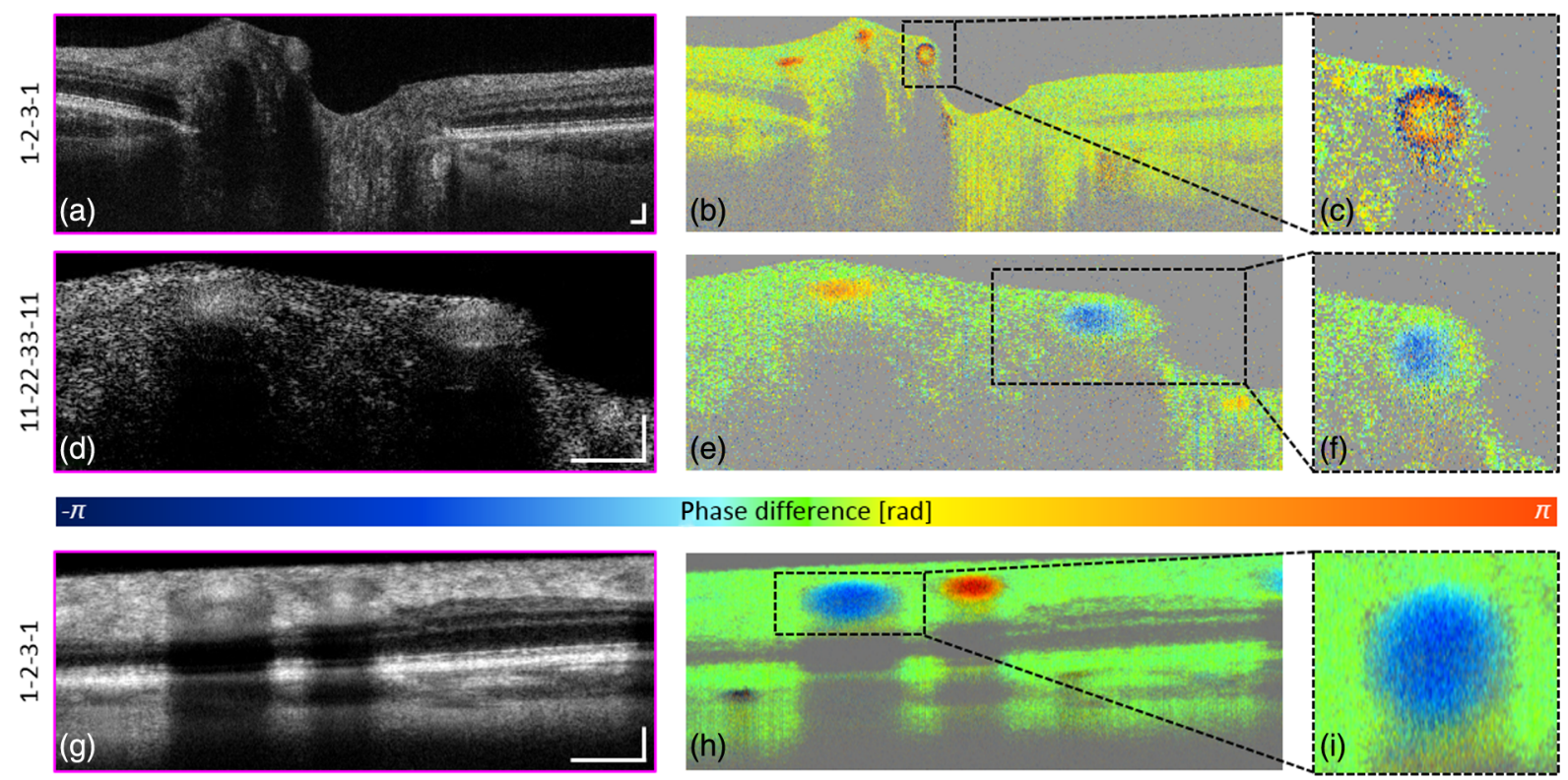

\section{Phase difference [rad]}
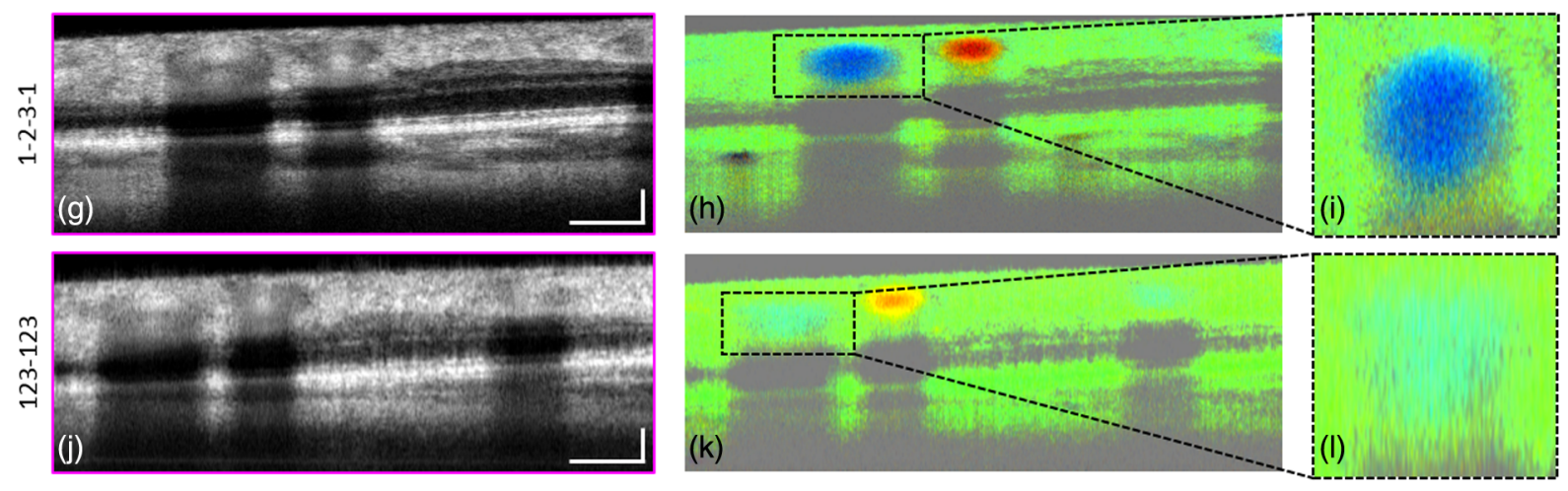

Fig. 5 In vivo qualitative Doppler evaluation and comparison of image data of the three different switching schemes (only channel-2). Linear scan trough ONH using 1-2-3-1 scheme (single-frame): (a) intensity, (b) Doppler tomogram, (c) zoom-in of (b). Linear scan at same retinal location recorded with reduced field of view using 11-22-33-11 scheme (single-frame): (d) Intensity, (e) Doppler tomogram, (f) zoom-in of (e). Linear scan superior to $\mathrm{ONH}$ using 1-2-3-1 scheme (cardiac cycle averaged-seven B-scans): (g) intensity, (h) Doppler tomogram, (i) zoom-in of (h). Linear scan at same retinal location using 123-123 scheme (cardiac cycle averaged-seven B-scans): (j) intensity, (k) Doppler tomogram, (I) zoom-in of (k). Scale bars: $100 \mu \mathrm{m}$. 


\subsection{In Vivo Retinal Blood Flow Experiments}

After the successful in vitro demonstration of the different switching schemes, in vivo RBF measurements were conducted. Figure 5 shows the challenge of determining suitable Doppler frequency intervals for retinal vessels with different axial flow velocity components. The optimum maximum unambiguously determinable Doppler frequency should provide sufficient difference between static and moving tissue components; however, without exceeding the limits of the $-\pi / \pi$ interval, which would cause phase wrapping artifacts. Phase wrapping artifacts obscure the parabolic-shaped velocity profile within a retinal vessel and thus usually prevent or at least heavily complicate exact flow quantification.

The different switching schemes provide a means to adapt the unambiguous $-\pi / \pi$ range to an extended range of flow velocities. Figures 5(a)-5(c) show a single-frame linear scan through the $\mathrm{ONH}$ (where usually high axial velocity components occur) of one of the three channels (channel-2) using the inter-A-scan switching scheme (1-2-3-1; maximum unambiguously determinable Doppler frequency $33.3 \mathrm{kHz}$ ). In the Doppler tomogram [Fig. 5(b)] and especially in its zoom-in [Fig. 5(c)], strong phase wrapping artifacts are obvious in the displayed artery. Figures 5(d)-5(f) show the same retinal location (reduced field of view) using the double inter-A-scan switching scheme (11-22-33-11; maximum unambiguously determinable Doppler frequency $100 \mathrm{kHz}$ ). In Figs. 5(e) and 5(f), the same artery does not show phase wrapping artifacts for the same phase of the cardiac cycle, enabling artifact free velocity measurements. Figures 5(g)-5(i) were again recorded with the 1-2-3-1 scheme, whereas Figs. 5(j)-5(1) were recorded with the intra-A-scan switching scheme (123-123; maximum unambiguously determinable Doppler frequency $100 \mathrm{kHz}$ ). In both cases, the images of a linear scan superior to the $\mathrm{ONH}$ are averaged over a full cardiac cycle (seven B-scans). Here, the zoom-ins of the Doppler tomograms highlight a large vein. As no phase wrapping artifacts occur for the 1-2-3-1 scheme [Fig. 5(j)], this scheme is the better alternative in comparison with the 123-123 scheme [Fig. 5(1)], where the vein is hardly distinguishable from the surrounding static retinal tissue.

In addition to the purely qualitative illustration of Fig. 5, quantitative measurements were performed in three eyes of healthy volunteers-cf. Figures 6, 7, and Table 2. One major artery at a similar retinal location superior to the $\mathrm{ONH}$ in each eye was imaged and evaluated regarding its 3-D velocity vectors.

For every artery, the averaged Doppler tomogram of a full cardiac cycle was evaluated and each measurement was repeated five times per switching scheme (same four schemes as for the in vitro experiments in Sec. 3.1). Figure 6 shows the measurement results graphically. Figures 6(a), 6(c), and 6(e) show fundus photos and a zoom-in of the ROI including an indication of the measurement location (black horizontal line). The orientation of the black arrow indicates the manually determined flow orientation within the fundus image of the respective artery at the measurement location, which serves as a ground truth for comparison with the measurement results. Unfortunately, a ground truth for the measured $\vec{v}_{\text {abs }}$ does not exist. Figures 6(b), 6(d), and 6(f) display the measurement results of the respective switching schemes in terms of a black arrow on top of a grayscale fundus photo. The arrow's orientation indicates the measured angle of flow $\delta$ in the en-face plane, whereas the length of the arrow is proportional to the measured $\vec{v}_{\text {abs }}$.

Table 2 lists the mean value and the coefficient of variation $(\mathrm{COV})$ for the measured absolute flow velocity $\left(\vec{v}_{\mathrm{abs}}\right)$ as well as the orientation of the velocity vector in the en-face plane $(\delta)$ per subject. The indicated values represent mean velocity values over the parabolic shaped vessel profiles.

Except for the two cases of the 1-2-3-1 scheme, measurement of subject 1 and subject 3 the measured $\vec{v}_{\text {abs }}$ and $\delta$ seem to be in good agreement among the individual switching schemes. Also, the measured orientation of flow is in very good agreement with the manually determined ground truth. The aforementioned two exceptions can be attributed to severe phase wrapping artifacts, which totally distort the measurement results. As no phase wrapping artifacts were present in the measurement results of the 1-23-1 scheme for subject 2, the length and orientation of the black arrow are in good agreement with the measurements of the other switching schemes.

Figure 7 shows confirmation of the observed phase wrapping artifacts in the measurements of subject 1 (left column) in comparison with subject 2 (right column). Here, the two switching schemes 1-2-3-1 and 123-123 are qualitatively compared between both subjects. Figures 7(a) and 7(c) show the full cardiac cycle averaged Doppler tomograms of the three channels for scheme 1-2-3-1 and Figs. 7(b) and 7(d) for scheme 123123 of subject 1 and 2, respectively. Figure 7(a) shows heavy

Table 2 Quantitative results of the in vivo absolute RBF velocity measurements using four different switching schemes in three eyes of three healthy volunteers. Five measurements per switching schemes and per eye where evaluated and mean and COV are listed. For every measurement, all B-scans within one cardiac cycle were averaged.

\begin{tabular}{|c|c|c|c|c|c|c|c|c|c|}
\hline & & \multicolumn{2}{|c|}{$1-2-3-1$} & \multicolumn{2}{|c|}{$11-22-33-11$} & \multicolumn{2}{|c|}{$123-123$} & \multicolumn{2}{|c|}{ Reduced 123-123 } \\
\hline & & Mean & COV\% & Mean & COV\% & Mean & $\mathrm{COV} \%$ & Mean & COV\% \\
\hline Subject 1 & $V_{\mathrm{abs}}[\mathrm{mm} / \mathrm{s}]$ & 11.5 & 27.6 & 16.1 & 11.4 & 14.0 & 12.8 & 14.8 & 7.7 \\
\hline$\delta=251 \mathrm{deg}$ & $\delta$ [deg] & 134.5 & 30.4 & 253.4 & 4.3 & 246.5 & 3.3 & 246.8 & 4.4 \\
\hline Subject 2 & $V_{\text {abs }}[\mathrm{mm} / \mathrm{s}]$ & 13.5 & 11.5 & 10.9 & 7.2 & 13.2 & 3.6 & 11.3 & 27.4 \\
\hline$\delta=253 \mathrm{deg}$ & $\delta$ [deg] & 252.1 & 1.2 & 248.3 & 4.0 & 257.4 & 1.7 & 251.7 & 3.6 \\
\hline Subject 3 & $V_{\text {abs }}[\mathrm{mm} / \mathrm{s}]$ & 8.0 & 37.8 & 13.5 & 9.7 & 13.5 & 8.7 & 13.8 & 11.8 \\
\hline$\delta=247 \mathrm{deg}$ & $\delta$ [deg] & 187.3 & 29.6 & 260.7 & 1.5 & 249.7 & 1.3 & 256.3 & 3.1 \\
\hline
\end{tabular}



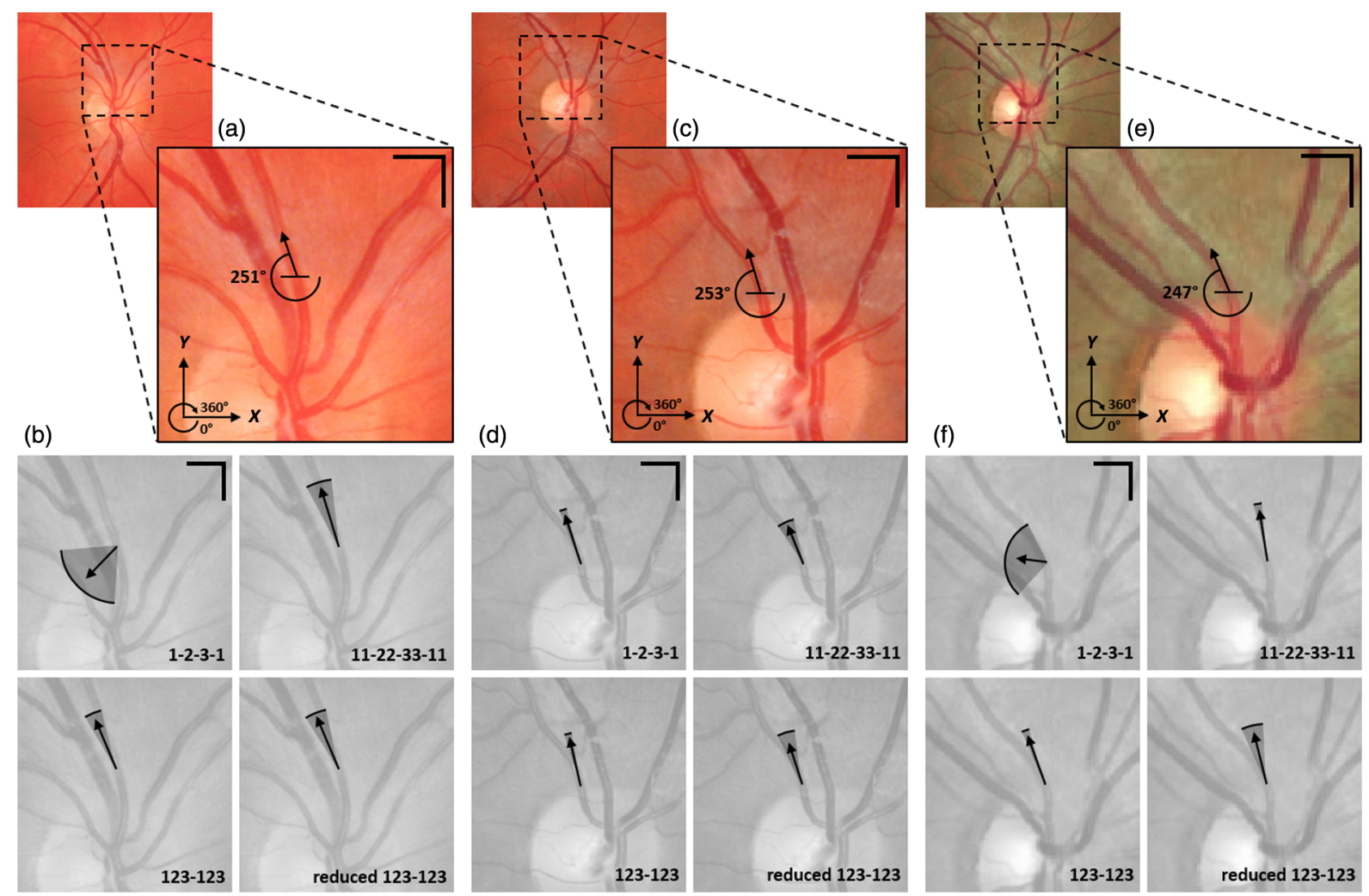

Fig. 6 In vivo absolute RBF velocity measurements using time-encoded multichannel OCT and different switching schemes in three eyes of three healthy volunteers. (a) and (b) subject 1, (c) and (d) subject 2 , (e) and (f) subject 3. (a), (c), (e) Fundus photos and zoom-in of respective ROls including indications of measurement location as well as manual angle determination of the respective artery. (b), (d), (f) Graphically illustrated measurement results of Table 2 for the four discussed switching schemes. The black arrow indicates the measured orientation of blood flow $(\delta)$ as well as the absolute flow velocity (length of arrow) projected onto the fundus plane. The shaded angular segments indicate \pm one STD of the measured angular orientation of flow $(\delta)$. The distance from arrow tip to the border of the angular segment indicates + one STD of the absolute flow velocity. Scale bars: $500 \mu \mathrm{m}$.

phase wrapping artifacts in channel-2, which are not visible in Fig. 7(b). Figures 7(c) and 7(d) do not show phase wrapping artifacts. However, the averaging over a full cardiac cycle might conceal phase wrapping artifacts arising only at specific phases of the cardiac cycle (i.e., the systolic phase). Thus, Figs. 7(f)-7(i) show time-resolved sequences of channel-2 for subject 1 and channel-1 for subject 2 of the above averages of Figs. 7(a)-7(d). The single-frame sequences explicitly demonstrate why the 1-2-3-1 scheme measurements of subject 1 are distorted in comparison with the ones of subject 2 . The presence of heavy phase wrapping artifacts during the systolic phase of the cardiac cycle is easily noticeable.

\section{Discussion}

Time-encoded multichannel OCT offers the possibility to enable multiple active channel operation at full illumination power quasisimultaneously. The integration of high-speed fiber optical switches, which was tested to not affect the intrinsic phase stability of the instrument, allows for switching of subsample arms and thus the incident illumination angle at repetition rates of up to several hundred $\mathrm{kHz}$. The enhanced signal quality due to increased illumination power especially benefits DOCT investigations because of decreased phase noise. However, this advantage comes at the cost of a reduced imaging speed and thus a reduced dynamic range for DOCT velocity measurements. By the introduction of adaptable illumination channel switching schemes, the reduced velocity range could be extended back to the original one, which is limited by the sweep rate of the TLS only.

Furthermore, the principle of time encoding reduces the hardware complexity of the system with the exception of the sample arm in comparison with our earlier work. ${ }^{9}$ Only one light source, one interferometer, one balanced detection unit, and one DAQ board are needed.

In comparison with our previous APPLE approach, ${ }^{11}$ the detected signal of the three channels does not result from an identical backscattering origin. Also other (nontime encoded) active multichannel OCT instruments might suffer from small lateral offsets of the focal spots of their individual beams due to optical aberrations introduced by the sample. ${ }^{9,33}$ Timeencoded multichannel OCT includes an additional source of possible lateral misalignment. Using continuous instead of step-wise scanning (which is impossible to achieve at the high imaging speeds used), the sequential recording results in slightly different scanning mirror positions for successive Ascans among the channels. However, when comparing the images of the three channels, these deviations were found to be negligible as long as a sufficiently high sampling is maintained. 


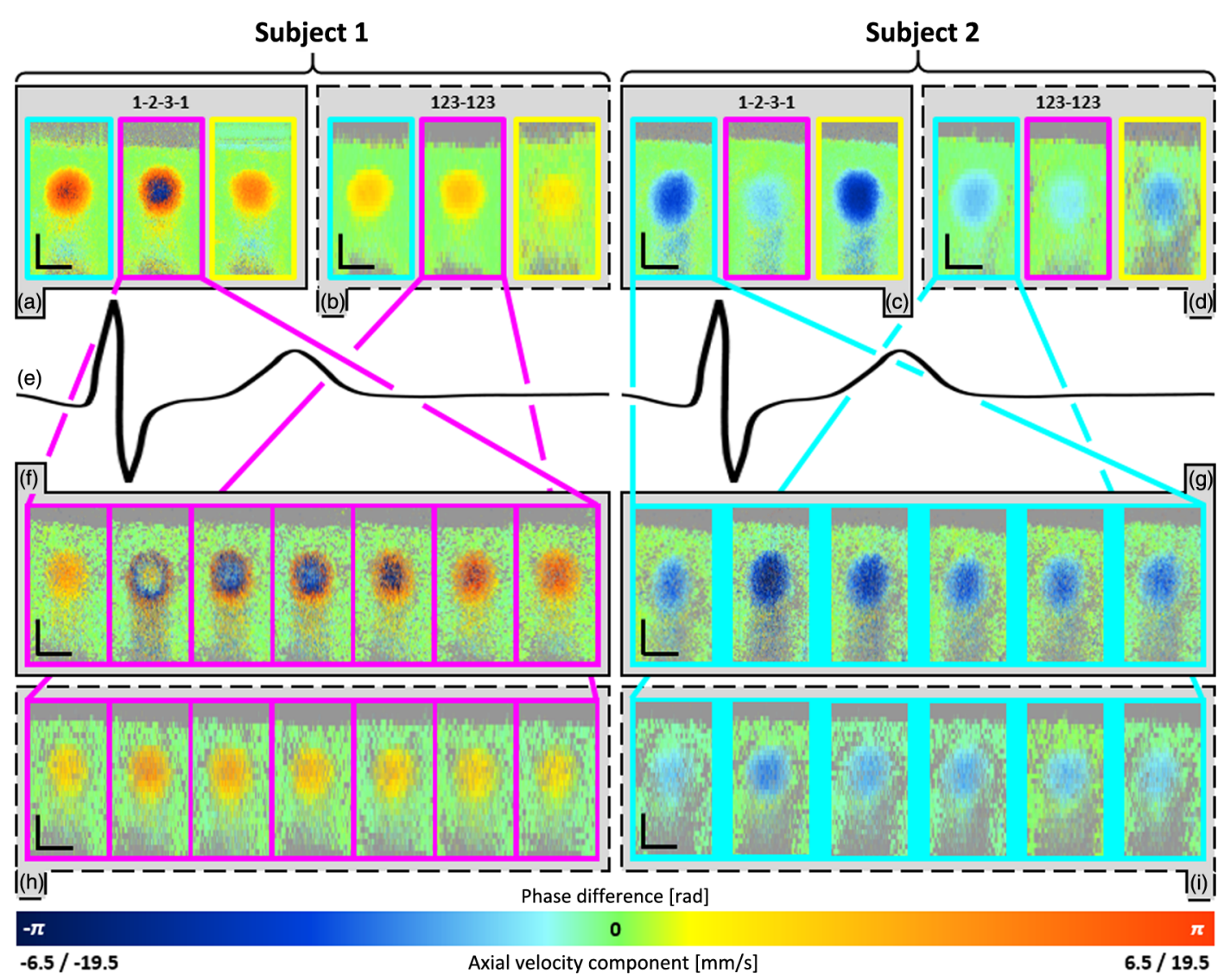

Fig. 7 Doppler tomogram comparison of the in vivo measurement results of Table 2 and Fig. 6 of the 1-23-1 and 123-123 switching scheme of subject 1 (left column) and subject 2 (right column). Cardiac cycle average (seven B-scans) of the same retinal location (subject 1) using the 1-2-3-1 scheme (a) and the 123-123 scheme (b). Cardiac cycle average (six B-scans) of the same retinal location (subject 2) using the 1-2-3-1 scheme (c) and the 123-123 scheme (d). (e) Simplified generic illustration of an ECG-signal trace. Single-frame images recorded at different phases of the cardiac cycle of the same retinal location (subject 1) using the 1-2-3-1 scheme (f) and the 123-123 scheme (h). Single-frame images recorded at different phases of the cardiac cycle of the same retinal location (subject 2) using the 1-2-3-1 scheme (g) and the 123-123 scheme (i). Scale bars: $100 \mu \mathrm{m}$.

Concerning the 123-123 switching scheme, the axial resolutions of the individual channels differ slightly from each other due to different central wavelengths for the equally sized spectral intervals. Also, the axial velocity component that is determined by DOCT is affected in the same way. However, these expected minor differences were regarded negligibly.

Additionally, the time interval in between a change of light transmission paths from output 1 to output 2 of an optical switch has direct consequences for the 123-123 switching scheme and was therefore investigated. The longer this time interval, the more spectral sample points would be rendered unusable for intra-A-scan switching. The manufacturer only specifies a socalled "response time (rise, fall)," which corresponds to the time interval in between the start of the electronical trigger signal sent to the optical switch and the end of the change of light transmission paths. This interval is specified to be in the range of 30 to $300 \mathrm{~ns}$. However, the time interval in between a change of light transmission paths is much shorter as the specified delay includes all necessary electronic signal processing. We were unable to directly measure the short time-delay ourselves, which is why an indirect verification whether the referred time interval was short enough to not render any spectral sample points unusable was obtained. Thus, we performed experiments where the individual components of a spectral sweep belonging each to one of the three channels were cropped at the edges by 1 , 2, 3, and more spectral sample points. Nevertheless, besides a reduction in axial resolution, no further effect was observed. Hence, we concluded that the actual switching time was below the balanced detection sampling limit of $200 \mathrm{MHz}$ (corresponding to intervals of $5 \mathrm{~ns}$ ). To obtain the best possible axial resolution, no spectral sample points were sacrificed for the measurements reported here. The smooth transitions from one to the next spectral interval observable in Fig. 2(e) show the above-discussed characteristic.

A drawback regarding the 123-123 switching scheme is the sensitivity penalty of $\sim 2 \mathrm{~dB}$ of the first spectral interval in comparison with the latter two. As apparent in Fig. 2(b), the amplitude of the modulation in the first interval is reduced. As this is at least partly caused by a wavelength dependence/imbalance of incorporated system components (e.g., fiber couplers), future integration into enhanced components supporting a broader wavelength bandwidth might mitigate this issue. Additionally, the laser sweep's nonuniform intensity distribution may also contribute to the observed sensitivity penalty.

Like other digital spectral splitting approaches, ${ }^{34}$ our hardware spectral splitting technique (i.e., the 123-123 switching 
scheme) also theoretically reduces measurement sensitivity according to SS-OCT theory. ${ }^{35}$ We observed a somewhat reduced image quality in the images recorded with the 123123 switching scheme, due to the combined effect of decreased axial resolution and reduced sensitivity. This quality reduction is a drawback of intra-A-scan switching and has to be considered as a trade-off versus increased imaging speed.

Another point of potential future system improvement concerns the linear scanning pattern. For a smooth linear scan, the 2-D MEMS scanner only supports the same number of A-scans for forward- and back-scan. If, instead of a triangular pattern a sawtooth pattern (featuring a reduced number of A-scans for the back-scan) is applied, the mirror shows irregular and nonuniform movement. As data acquisition was restricted to the forward-scan, the imaging time could be further reduced by a factor of two by additionally recording while back-scanning.

The other way of imaging time reduction presented in this work capitalizes from the difference in the number of provided phase-difference A-scans for the 11-22-33-11 and the 123-123 (six-times as many as for the 11-22-33-11 scheme) switching scheme for the same imaging duration. The reduced 123-123 scheme (Figs. 5-7) is six-times faster and still shows reasonable results [standard deviation (STD) mostly a little higher than for the other switching schemes in Table 2], even though the number of phase-difference pixels available for Doppler evaluation is reduced.

The investigation of RBF is important, especially for diseases such as glaucoma and diabetic retinopathy. Alterations of RBF seem to be in close correlation with the development of these pathologic disorders. ${ }^{36-38}$ Our time-encoded multichannel OCT prototype might prove a valuable tool for diagnosis and monitoring of these diseases.

Even though the results presented here exclusively focus on flow phantom and RBF investigations using DOCT, our technology potentially offers a variety of possible benefits for OCT subfields ranging from angular speckle reduction to OCT-based angiography. In particular, the recently introduced field of multidirectional $\mathrm{OCT}^{33}$ would profit from an enhanced sensitivity as well as from a flexible channel alignment. In addition, an application of time-encoded multichannel OCT in other medical fields, besides ophthalmology, such as dermatology or neurology would potentially prove to be beneficial.

\section{Disclosures}

The authors declare that there are no conflicts of interest related to this article.

\section{Acknowledgments}

This work was supported by the Austrian Science Fund (FWF; grant number: P26553-N20), the Christian Doppler Laboratory (CDL; grant: OTPIMA). We further wish to acknowledge Wolfgang Trasischker, Lara M. Wurster, Marco Augustin, Rainer Leitgeb, Marie Laslandes, Matias Salas, Richard Haindl, Laurin Ginner, Danielle J. Harper, Pablo Eugui, Antonia Lichtenegger, and Sylvia Desissaire for their assistance and helpful discussions.

\section{References}

1. R. A. Leitgeb et al., "Doppler optical coherence tomography," Prog. Retinal Eye Res. 41(100), 26-43 (2014).
2. Y. Wang et al., "In vivo total retinal blood flow measurement by Fourier domain Doppler optical coherence tomography," J. Biomed. Opt. 12(4), 041215 (2007).

3. R. Michaely et al. "Vectorial reconstruction of retinal blood flow in three dimensions measured with high resolution resonant Doppler Fourier domain optical coherence tomography," J. Biomed. Opt. 12(4), 041213 (2007).

4. H. Wehbe et al., "Automatic retinal blood flow calculation using spectral domain optical coherence tomography," Opt. Express 15(23), 1519315206 (2007).

5. C. Blatter et al., "Dove prism based rotating dual beam bidirectional Doppler OCT," Biomed. Opt. Express 4(7), 1188-1203 (2013).

6. V. Doblhoff-Dier et al., "Measurement of the total retinal blood flow using dual beam Fourier-domain Doppler optical coherence tomography with orthogonal detection planes," Biomed. Opt. Express 5(2), 630-642 (2014).

7. N. V. Iftimia et al., "Dual-beam Fourier domain optical Doppler tomography of zebrafish," Opt. Express 16(18), 13624-13636 (2008).

8. R. M. Werkmeister et al., "Bidirectional Doppler Fourier-domain optical coherence tomography for measurement of absolute flow velocities in human retinal vessels," Opt. Lett. 33(24), 2967-2969 (2008).

9. W. Trasischker et al., "In vitro and in vivo three-dimensional velocity vector measurement by three-beam spectral-domain Doppler optical coherence tomography," J. Biomed. Opt. 18(11), 116010 (2013).

10. International Electrotechnical Commission, "Safety of laser productspart 1: equipment classification and requirements," IEC-60825-1:2014 (2014).

11. A. Wartak et al., "Active-passive path-length encoded (APPLE) Doppler OCT," Biomed. Opt. Express 7(12), 5233-5251 (2016).

12. T. Klein et al., "Joint aperture detection for speckle reduction and increased collection efficiency in ophthalmic MHz OCT," Biomed. Opt. Express 4(4), 619-634 (2013).

13. A. Wartak et al., "Sequential multi-channel OCT in the retina using high-speed fiber optical switches," Proc. SPIE 10416, 1041607 (2017).

14. J. Ai and L. V. Wang, "Spectral-domain optical coherence tomography: removal of autocorrelation using an optical switch," Appl. Phys. Lett. 88(11), 111115 (2006).

15. H. Wang, Y. Pan, and A. M. Rollins, "Extending the effective imaging range of Fourier domain optical coherence tomography using a fiber optic switch," Opt. Lett. 33(22), 2632-2634 (2008).

16. S.-W. Lee, H.-W. Jeong, and B.-M. Kim, "High-speed spectral domain polarization-sensitive optical coherence tomography using a single camera and an optical switch at $1.3 \mu \mathrm{m}$," J. Biomed. Opt. 15(1), 010501 (2010).

17. O. P. Kocaoglu et al., "Adaptive optics optical coherence tomography at $1 \mathrm{MHz}$," Biomed. Opt. Express 5(12), 4186-4200 (2014).

18. A.-H. Dhalla, K. Shia, and J. A. Izatt, "Efficient sweep buffering in swept source optical coherence tomography using a fast optical switch," Biomed. Opt. Express 3(12), 3054-3066 (2012).

19. M. G. Hyeon et al., "Spectral domain optical coherence tomography with balanced detection using single line-scan camera and optical delay line," Opt. Express 23(18), 23079-23091 (2015).

20. M. Servin et al., "Phase unwrapping through demodulation by use of the regularized phase-tracking technique," Appl. Opt. 38(10), 1934-1941 (1999).

21. K. J. Chalut, W. J. Brown, and A. Wax, "Quantitative phase microscopy with asynchronous digital holography," Opt. Express 15(6), 3047-3052 (2007).

22. C. K. Hitzenberger et al., "Differential phase measurements in lowcoherence interferometry without $2 \pi$ ambiguity," Opt. Lett. 26(23), 1864-1866 (2001).

23. H. C. Hendargo et al., "Synthetic wavelength based phase unwrapping in spectral domain optical coherence tomography," Opt. Express 17(7), 5039-5051 (2009).

24. Y. Xu et al., "Filtering for unwrapping noisy Doppler optical coherence tomography images for extended microscopic fluid velocity measurement range," Opt. Lett. 41(17), 4024-4027 (2016).

25. S. Xia et al., "Robust phase unwrapping for phase images in Fourier domain Doppler optical coherence tomography," J. Biomed. Opt. 22(3), 036014 (2017).

26. I. Grulkowski et al., "Scanning protocols dedicated to smart velocity ranging in spectral OCT," Opt. Express 17(26), 23736-23754 (2009). 
27. P. Meemon and J. P. Rolland, "Swept-source based, single-shot, multidetectable velocity range Doppler optical coherence tomography," Biomed. Opt. Express 1(3), 955-966 (2010).

28. X. Shu et al., "Spectroscopic Doppler analysis for visible-light optical coherence tomography," J. Biomed. Opt. 22(12), 121702 (2017).

29. R. Haindl et al., "Three-beam Doppler optical coherence tomography using a facet prism telescope and MEMS mirror for improved transversal resolution," J. Mod. Opt. 62(21), 1781-1788 (2015).

30. R. Haindl et al., "Total retinal blood flow measurement by three beam Doppler optical coherence tomography," Biomed. Opt. Express 7(2), 287-301 (2016).

31. M. Hafez, T. Sidler, and R.-P. Salathé, "Study of the beam path distortion profiles generated by a two-axis tilt single-mirror laser scanner," Opt. Eng. 42(4), 1048-1057 (2003).

32. C. K. Hitzenberger, "Optical measurement of the axial eye length by laser Doppler interferometry," Invest. Ophthalmol. Visual Sci. 32(3), 616-624 (1991).

33. A. Wartak et al., "Multi-directional optical coherence tomography for retinal imaging," Biomed. Opt. Express 8(12), 5560-5578 (2017).

34. Y. Jia et al., "Split-spectrum amplitude-decorrelation angiography with optical coherence tomography," Opt. Express 20(4), 4710-4725 (2012).

35. M. Choma et al., "Sensitivity advantage of swept source and Fourier domain optical coherence tomography," Opt. Express 11(18), 2183 2189 (2003).

36. J. Flammer et al., "The impact of ocular blood flow in glaucoma," Prog. Retinal Eye Res. 21(4), 359-393 (2002).

37. J. T. Durham and I. M. Herman, "Microvascular modifications in diabetic retinopathy," Curr. Diabetes Rep. 11(4), 253-264 (2011).

38. D. Schmidl, G. Garhofer, and L. Schmetterer, "The complex interaction between ocular perfusion pressure and ocular blood flow-relevance for glaucoma," Exp. Eye Res. 93(2), 141-155 (2011).

Andreas Wartak received his BSc degree in technical physics and his MSc degree in biomedical engineering from Vienna University of Technology, Austria. Currently, he is a doctoral student in medical physics at the Center for Medical Physics and Biomedical Engineering at the Medical University of Vienna, Austria. His research focuses on OCT, in particular on the improvement and development of multichannel techniques for application in ophthalmology with emphasis on retinal imaging.

Florian Beer received his BSc and MSc degrees from Vienna University of Technology in technical physics. Currently, he is enrolled as a doctoral student at Vienna University of Technology and his work is carried out at the Center for Medical Physics and Biomedical Engineering at the Medical University of Vienna. His research interests include polarization-sensitive OCT (PS-OCT), in particular for anterior eye imaging.

Bernhard Baumann is an associate professor at the Center for Medical Physics and Biomedical Engineering at the Medical University of Vienna. After receiving his PhD from the Medical University of Vienna, he joined the Research Laboratory of Electronics at the Massachusetts Institute of Technology, Cambridge, MA, and the New England Eye Center at Tufts University, Boston, MA, for a joint postdoc fellowship, before returning to Vienna.

Michael Pircher is a professor of medical physics at the Center for Medical Physics and Biomedical Engineering at the Medical University of Vienna and associate editor of Biomedical Optics Express (BOE). He received his PhD from Vienna University of Technology in Technical Sciences and completed his habilitation in medical physics at the Medical University of Vienna.

Christoph K. Hitzenberger is a professor of medical physics and vice-chair of the Center for Medical Physics and Biomedical Engineering at the Medical University of Vienna, and editor-in-chief of BOE. He works in biomedical optics since 1987 and is one of the pioneers of low coherence ocular biometry and OCT. He is a fellow of SPIE and OSA. His pioneering contributions to OCT were awarded with the Russ Prize of the National Academy of Engineering in 2017. 